Cahiers $d u$ MONDE RUSSE

\section{Cahiers du monde russe}

Russie - Empire russe - Union soviétique et États indépendants

\title{
Georges Dulac, Sergueï Karp, éds., Les Archives de l'Est et la France des Lumières
}

\section{Francine-Dominique Liechtenhan}

\section{(2) OpenEdition \\ Journals}

Édition électronique

URL : https://journals.openedition.org/monderusse/7012

DOI : 10.4000/monderusse. 7012

ISSN : $1777-5388$

Éditeur

Éditions de l'EHESS

Édition imprimée

Date de publication : 28 décembre 2008

Pagination : 838-842

ISBN : 978-2-7132-2197-2

ISSN : $1252-6576$

Référence électronique

Francine-Dominique Liechtenhan, « Georges Dulac, Sergueï Karp, éds., Les Archives de l'Est et la France des Lumières ", Cahiers du monde russe [En ligne], 49/4 | 2008, mis en ligne le 24 décembre 2009, consulté le 03 septembre 2022. URL : http://journals.openedition.org/monderusse/7012 ; DOI : https://doi.org/10.4000/monderusse. 7012

Ce document a été généré automatiquement le 3 septembre 2022.

Tous droits réservés 


\title{
Georges Dulac, Sergueï Karp, éds., Les Archives de l'Est et la France des Lumières
}

\author{
Francine-Dominique Liechtenhan
}

\section{RÉFÉRENCE}

Georges DULAC, Sergueï KARP, éds., Les Archives de l'Est et la France des Lumières.

Ferney-Voltaire : Centre international d'étude du XVIII siècle, 2007, 2 vols., 870 p.

1 Voici un ouvrage qu'attendaient depuis longtemps les chercheurs intéressés aux relations franco-russes. Il concerne en particulier ceux, débutants ou non russophones, qui connaissent mal les méandres des archives de l'ex-URSS. Les Archives de l'Est et la France des Lumières, publiées sous la direction de Georges Dulac et Sergueï Karp, assistés par une pléiade de spécialistes du XVIII ${ }^{e}$ siècle, représentent un outil de travail important, dans l'attente que l'École des chartes publie ses propres inventaires des fonds français en Russie.

2 Le premier volume se lit comme un guide. Il cerne une partie considérable des archives situées dans la Fédération de Russie, en Ukraine, Estonie, Lituanie ou Finlande et décrit les fonds les plus importants relatifs à la civilisation française. Les auteurs ont dû écarter la Pologne, ancien Duché de Varsovie, pour les raisons que l'on devine, mais aussi la Sibérie et les parties asiatiques de l'immense empire. Leur objectif est de fournir des exemples susceptibles de donner une image "précise, mais jamais complète» des principaux dépôts d'archives hébergés en Russie. Des renseignements pratiques (adresse, courriel, site, nom des directeurs et conservateurs) précèdent l'histoire de ces archives : acquisition, établissement des inventaires et fusion de certains dépôts dont les plus anciens datent $d u \mathrm{XVIII}^{\mathrm{e}}$ siècle. Les riches indications bibliographiques situées en notes infrapaginales permettent à l'utilisateur de s'orienter. Quelques petites erreurs (par exemple, p. 241, Favier ne reste pas en Russie de 1760 à 1763, mais n'arrive qu'en 
février 1761 pour repartir en octobre de la même année) n'obèrent pas la valeur de cet instrument de travail. La trajectoire de certains fonds, notamment privés, et l'histoire des collectionneurs n'ont pas été oubliées. Petr P. Dubrovskij, secrétaire à l'ambassade russe à Paris, profita des émois révolutionnaires pour acheter une partie des archives de la Bastille, dont des correspondances et des livres saisis par la police française. À la même époque, le comte Aleksandr R. Voroncov traqua des documents diplomatiques pour lesquels il dépensa 2400 livres tournois. Les collectionneurs se succédèrent au xiX siècle avec Grigorij V. Orlov ou Aleksandr I. Turgenev entre autres. Les Soviétiques s'empressèrent à leur tour de compléter leurs collections, les conservateurs de l'Institut du marxisme-léninisme s'avérant de fervents amateurs de documents anciens. Georges Dulac et Sergueï Karp ont l'immense mérité d'avoir reconstitué des séries perdues grâce à des minutes achetées jadis par des aristocrates russes afin d'enrichir leurs propres collections. Les maitres d'œuvre ont tenu compte de toutes les catégories de textes qui peuvent contribuer à illustrer les relations entre les deux pays: lettres autographes, correspondances, projets politiques, manuscrits littéraires, traductions et simples commandes ou factures de "galanteries », pour ne nommer que les plus importants. Ils n'ont pas oublié les hommes, écrivains célèbres, aventuriers ou simples ouvriers qui voyagèrent en Russie, acteurs plus ou moins importants des relations culturelles, dont les traces s'évanouissent souvent dans le néant. Les diplomates et leurs secrétaires, conseillers ou consuls, dont certains pesèrent pourtant de tout leur poids sur les relations culturelles franco-russes, nous paraissent trop effacés dans ce cortège de personnalités centré sur les sciences et les lettres.

3 Le deuxième volume pourrait se lire séparément du « guide ». Il s'agit d'une anthologie de textes inédits que les auteurs ont retenus comme exemplaires de la présence française dans la Russie des Lumières. Comme il se doit, il se clôt sur de nouveaux instruments de travail, à savoir un index et une bibliographie, dont on peut déplorer certaines lacunes : par exemple l'ouvrage magistral de Karl Stählin, Aus den Papieren Jacobs von Stählin, ein biographischer Beitrag zur deutsch-russischen Geschichte des 18. Jahrhunderts (Königsberg: Osteuropa-Verlag, 1926, 457 p.) ou, plus récent, le recueil publié par Jean-Pierre Poussou en 2004, L'Influence française en Russie au XVIII' siècle (P. : Institut d'études slaves, Presses de l'Université Paris-Sorbonne, 733 p.).

Ce volume est divisé en quatre parties. La première est consacrée à l'Académie des sciences de Saint-Pétersbourg et à certains de ses membres : les Euler, père et fils, Stählin et Rousset de Missy. Le Supplément à l'Encyclopédie était alors un sujet de débat majeur. L'Académie impériale russe fut sollicitée de lui fournir des éléments complémentaires, mais se heurta à son secrétaire, Johann Albrecht Euler, francophobe invétéré. Les contacts avec des libraires suisses sont évoqués dans deux documents importants. Une lettre du baron de Tchoudy montre comment furent rassemblés certains matériaux destinés à aider Voltaire pour son Histoire de la Russie sous Pierre le Grand.

5 Le deuxième volet concerne les relations des cercles philosophiques français avec la Russie. Il jette une lumière nouvelle sur le rôle de Diderot dans la vie culturelle russe et ses contacts personnels avec certaines élites. Installé à Spa, l'abbé Raynal, fustigé par le Parlement de Paris pour son Histoire des deux Indes, se rapprocha de Sergej Rumjancev, qui chercha à l'aider à obtenir la protection de Catherine. D'autres passèrent par des tiers pour adresser messages ou écrits à la souveraine. Refroidi par l'attitude de cette dernière, le Napolitain Galiani préféra adresser ses propres lettres susceptibles d'intéresser Catherine II à Grimm. 
6 Le titre de la troisième partie, "L'Europe française », pourrait induire en erreur: on pense spontanément à la francophonie, élément fondateur de l'Europe des Lumières. Or les auteurs ont choisi les grands mouvements de pensée, pas exclusivement français par ailleurs. L'influence de la franc-maçonnerie, avec Gilbert Romme, y côtoie les grands débats autour de la Révolution de 1789. On y découvre un Aleksandr $\mathrm{I}^{\mathrm{er}}$ enclin aux idées radicales dans les années 1790. L'attitude mitigée d'une petite partie de la haute noblesse russe face au républicanisme se précise grâce à un « jacobin », en la personne du comte Pavel A. Stroganov, qui se hissa ultérieurement aux plus hauts rangs de l'administration. Le comte de Langeron et le duc de Richelieu, tous deux émigrés à l'Est, observèrent avec suspicion les antagonismes entre royalistes et modérés, sans épargner à ces derniers des épithètes plutôt crues. On doit au second des propos très intéressants sur les adversaires de Napoléon à la cour russe, réfractaires à tout rapprochement avec la France régicide.

7 Si ces trois volets relèvent d'une certaine cohérence thématique, on ne saurait en dire autant du quatrième choix de documents. Quelques lettres autographes (d'Alembert, Malesherbes, le diplomate sarde Triqueti et plusieurs pièces sur Montesquieu) sont néanmoins révélatrices de l'exceptionnelle diversité des fonds d'archives français dans la Fédération de Russie.

8 Il est certes très difficile d'effectuer une sélection de documents qui puisse satisfaire chercheurs et amateurs de littérature ou d'histoire. La critique est toujours facile, et, en fonction des écoles, un tel recueil pourrait prendre une tournure bien différente. Les adeptes d'une historiographie basée sur la longue durée, prônée par Fernand Braudel, regretteront que les auteurs, éminents spécialistes de la deuxième moitié du XVIII siècle, aient trop privilégié l'époque de Catherine II, la mieux connue en France. Certes, Voltaire dit en 1771 dans une «Épître » à cette impératrice que les Lumières venaient du Nord. Cela signifie-t-il vraiment qu'elles n'émergèrent que vers la deuxième moitié du XVIII ${ }^{e}$ siècle ? La question de la chronologie de cette France éclairée, dont les débuts sont souvent situés à la fin $\mathrm{du} \mathrm{xvII}^{\mathrm{e}}$ siècle, mérite d'être posée. Sur la base d'une telle définition, le règne de Pierre le Grand, ses contacts avec l'Académie des sciences de Paris et le bibliothécaire du Roi, l'abbé Bignon, s'intègrent dans cette problématique. D'innombrables textes hagiographiques publiés après la mort du "réformateur » ou " créateur » de la Russie moderne témoignent de la pertinence, dans ce cas, du concept certes artificiel de modernité. Dans l'euphorie russophile des philosophes, sa seconde épouse, une roturière, fut érigée en " Sémiramis du Nord ».

Seuls six documents, sur vingt-six, portent sur les décennies antérieures à 1762. Un seul concerne le règne d'Anna Ioannovna, les autres se référant aux années 1740-1750. Cette impératrice ne mérite certes pas l'épithète de monarque " éclairée ». Les dix années de son règne furent pourtant une période faste de l'Académie des sciences de SaintPétersbourg qui vit déjà des recrutements de Français, à des postes modestes il est vrai. Leur présence explique à sa manière une certaine francophobie ambiante chez les membres allemands de l'Académie impériale russe. Depuis plusieurs décennies, la Russie offrait un exceptionnel terrain d'accueil. À la suite de la révocation de l'Édit de Nantes, la régente Sophie avait accepté de recevoir des familles françaises huguenotes sur son territoire. Celles-ci s'installèrent d'abord dans le faubourg des étrangers à Moscou. Nombre d'entre elles, passablement russifiées, suivirent la cour dans la nouvelle capitale au début du xvIII ${ }^{e}$ siècle. On peut ainsi se demander comment cette minorité recevait les immigrants et quelle fut son influence sur les nouveaux venus, notamment quant aux réseaux, familiaux, religieux ou, en l'occurrence, maçonniques qu'elle leur offrait. 
10 Le second volume regorge ainsi de documents inédits savamment introduits et commentés; nous ne saurions tous les évoquer et nous arrêterons aux plus représentatifs à nos yeux. L'un des collaborateurs de ce volume, Vladimir Somov, présente deux dissertations de Voltaire sur le servage, conservées à la Bibliothèque nationale russe de Saint-Pétersbourg. Il s'agit de copies, établies par Jean-Louis Wagnière, le secrétaire du philosophe; l'attribution de ces textes, grâce à l'analyse méticuleuse de V. Somov, ne fait pas de doute. Ce dernier les présente avec un important développement, dûment annoté, sur l'histoire de la Société libre d'économie et sur l'histoire des fonds où se dissimulèrent ces papiers pendant plus de deux siècles. Alexandre Stroev décrit plusieurs documents issus des « Archives spéciales », consacrés à l'âge d'or de la franc-maçonnerie en Russie. Deux pièces que l'on croyait perdues pendant la Seconde Guerre mondiale viennent illustrer l'adhésion, difficile, de Charles Gilbert Romme à la loge maçonnique des Neuf Sœurs fondée en 1776. Le père du calendrier républicain fait aussi l'objet d'une recherche minutieuse menée par Alexandre Tchoudinov: le précepteur de Pavel A. Stroganov entreprit un "grand tour » avec son protégé et échoua en 1789-1790 en France. L'analyse des motivations politiques des deux hommes, de leurs divergences aussi, est complétée par quatre lettres entre le père, le précepteur et sa pupille russe qui allait entrer au Club des Jacobins! Parmi d'autres fleurons de ce deuxième volume, évoquons les deux "Mémoires de Ribeiro Sanchez sur la "civilisation" de la Russie ». Disciple de Hume, de Robertson et des physiocrates, Sanchez souligne l'importance de l'agriculture dans l'évolution des sociétés et s'adonne à une violente critique du servage, principale entrave à l'établissement d'une société civile. Ce texte d'une grande importance aurait mérité des commentaires plus étoffés de la part de son présentateur Georges Dulac. L'auteur portugais y analyse l'Église orthodoxe et dénonce l'influence néfaste du Saint-Siège. Un bref aperçu des relations entre les Églises, mais aussi du rapport de cet auteur israélite, expulsé de Russie pour ses pratiques en 1747, avec le christianisme aurait éclairé un texte pas réellement novateur, car Sanchez essaie, comme nombre de ses prédécesseurs, d'appliquer une théorie philosophique occidentale à une réalité russe, orientale car orthodoxe. Il analyse aussi la démographie, le système fiscal et les techniques de recrutement des serfs. Les notes consacrées à l'histoire économique et sociale du XVIII ${ }^{e}$ siècle restent succinctes, alors que l'on aurait facilement pu se dispenser de précisions géographiques, voire de commentaires répétitifs (notes 48,53 et 118). Les recherches d'Arcadius Kahan et Sergueï M. Troitski ne sauraient être oubliées dans un tel contexte, tout comme dans la bibliographie. Certaines tournures sont inhabiles : à la page 482, note 112, on lit: «Alexis, qui fut le premier souverain russe à s'intéresser à l'Occident [...] »; c'était oublier Ivan III, Ivan le Terrible et Boris Godunov et la présence importante d'étrangers, soit artisans, architectes ou militaires en Moscovie depuis la fin $\mathrm{du} \mathrm{XV}^{\mathrm{e}}$ siècle. Sans doute s'agit-il là d'un fâcheux raccourci dû à un problème technique qui aura échappé aux maîtres d'œuvre, tout comme certaines coquilles (p. 5 «l'ukrainiens »).

11 Malgré ces réserves dues à des conceptions historiographiques diverses, l'ouvrage de Georges Dulac et de Sergueï Karp garde toute sa valeur, dans l'attente que le grand projet d'inventaires des fonds français en Russie dirigé par l'Ecole des chartes soit réalisé ; les informations pratiques que Les Archives de l'Est fournissent, l'intérêt de certains documents inédits et l'excellence de la présentation de la majorité d'entre eux le rendent momentanément incontournable. 